\title{
Qualifizieren Sie sich mit der DHZ
}

\section{DHZ-FORTBILDUNGSPUNKTE:}

\section{Unterstiitzung durch viele Fachverbånde}

\author{
Viele Leser der DHZ sammeln DHZ-Fortbildungspunkte für das freiwillige \\ Fortbildungszertifikat für Heilpraktiker. Seit 2010 unterstützen der BDH \\ sowie weitere Fachverbände die Fobi-Punkte der DHZ als Nachweis für \\ kontinuierliche Fortbildung.
}

Heilpraktiker bilden sich sehr engagiert und regelmäßig fort ganz ohne Zwang und Pflicht, einfach weil sie wissen, dass sie dies ihren Patienten schuldig sind und eine Praxis sich heutzutage auch kaum anders erfolgreich führen ließe. Doch tatsächlich sind sie nach der Berufsordnung für Heilpraktiker $(\mathrm{BOH}$, Artikel 5) zur ständigen Fortbildung verpflichtet. Diese Fortbildung müssen sie zudem nachweisen - im Extremfall vor Gericht.

Ganz unabhängig davon ist eine kontinuierliche Qualifizierung heutzutage unerlässlich. Nicht nur Politiker und maßgebliche Vertreter des Gesundheitswesens fordern eine dokumentierte Qualitätssicherung. Moderne, aufgeklärte Patienten erwarten ebenfalls - völlig zu Recht - aktualisiertes Wissen und umfassende Kompetenz auf neuestem Stand von ihren Therapeuten.

\section{Fortbildungszertifikate - längst Alltag in vielen Medizin- berufen}

Viele medizinische Berufe haben inzwischen ein eingeführtes Fortbildungsprogramm, das kontinuierliches Weiterlernen fordert, dokumentiert, zertifiziert und nicht zuletzt auch das Ansehen des Zertifizierten steigert. Das CME-System (Continuing Medical Education) der Ärzte hat sich weltweit durchgesetzt. Aber auch Apotheker, pharmazeutisch-technische Assistenten, Pflegekräfte, Physiotherapeuten und einige Heilpraktikerverbände haben die Notwendigkeit der kontinuierlichen Fortbildung erkannt und inzwischen ihre eigenen Fortbildungszertifikate entwickelt. Gerade vor dem Hintergrund der Berufsstandssicherung ist der Nachweis qualifizierter Fortbildung von zentraler Bedeutung. So bietet z. B. der Bund Deutscher Heilpraktiker (BDH) gemeinsam mit anderen Fachverbänden ein freiwilliges Fortbildungszertifikat für Heilpraktiker an. Diese Institutionen nutzen die DHZFortbildungspunkte, um ihren Mitgliedern interaktive Fortbildung zu ermöglichen.

\section{DHZ-Punkte sammeln für das Zertifikat}

Das freiwillige Fortbildungszertifikat für Heilpraktiker des BDH und weiterer Fachverbände basiert im Wesentlichen auf dem oben beschriebenen generell anerkannten Fortbildungsmodell (CME) und garantiert so die Akzeptanz durch Öffentlichkeit, an- dere Gesundheitsberufe und die Politik. Die Grundeinheit dieser Fortbildungsaktivitäten ist der Fortbildungspunkt (FP). Dieser entspricht in der Regel einer abgeschlossenen Fortbildungsstunde (45 min). Für die erworbenen Fortbildungspunkte muss ein Nachweis erbracht werden. Punkte können z. B. entweder durch den Besuch von Fortbildungsveranstaltungen, Kongressen, Fachtagungen oder Workshops gesammelt oder über sogenannte interaktive Fachfortbildungen durch Internet, CD-ROM oder Fachzeitschriften erworben werden. Bei der interaktiven Fachfortbildung sind der Nachweis der Qualifizierung und die schriftliche Auswertung des Lernerfolgs eine Grundvoraussetzung für die Vergabe der Fortbildungspunkte.

Das Fortbildungszertifikat für Heilpraktiker wird ausgestellt, wenn Sie in drei Jahren 120 Fortbildungspunkte erworben, diese dokumentiert und einen entsprechenden Antrag auf Zertifizierung bei der Dokumentationsstelle des BDH gestellt haben. Davon können Sie maximal 12 Punkte pro Jahr für das Zertifikat mithilfe der interaktiven Fortbildung durch Fachzeitschriften sammeln (Infos unter www.bdh-online.de).

Das Fortbildungszertifikat steht allen Heilpraktikern offen, unabhängig von ihrer Verbandszugehörigkeit.

\section{Homöopathie-Zertifikat: Punkte erwerben mit der DHZ}

Die SHZ ist eine gemeinnützige, von Einzelinteressen unabhängige Stiftung des bürgerlichen Rechts.

Sie hat die Aufgabe, ein hohes Qualitätsniveau in der Ausund Weiterbildung von Homöopathen (Heilpraktikern und Ärzten) zu fördern und zu sichern, mit dem Ziel, Qualitätskriterien für eine klassisch homöopathische Behandlung in der Öffentlichkeit zu etablieren. Die umfassenden Qualifikationsrichtlinien definieren Inhalt und Umfang homöopathischer Ausbildungen (mindestens 550 Unterrichtseinheiten Kontaktstudium und 1250 Unterrichtseinheiten Heimstudium). Sie stellen sicher, dass die überprüften Therapeuten die klassische Homöopathie in Theorie und Praxis beherrschen. Für Homöopathen nach der Ausbildung ist neben einer Zertifikatsprüfung eine 3-jährige Supervisionszeit 


\section{DHZ-Fortbildungspunkte - Fragebogen}

\section{Es ist jeweils nur eine Aussage pro Auswahl korrekt. Bitte wählen Sie bei allen Aussagen die jeweils richtige. Die Aussagen beziehen sich auf die Artikel S. 14-19 und 20-22.}

\section{Welche Aussage trifft jeweils zu?}

\section{Auswahl 1}

a Traumatisierungen werden in der Regel durch unbekannte Personen verursacht.

b Das Wort „Trauma“ leitet sich vom griechischen „troin“ für „Traum“ ab.

c Traumatisierungen müssen nicht zwingend zu psychischen Erkrankungen führen.

d Die flexible innere Widerstandsfähigkeit gegenüber erhöhtem Stress nennt man Stringenz.

e Traumatisierungen geschehen in der Regel nur bis zum dritten Lebensjahr.

\section{Auswahl 2}

a Traumatisierungen aus einmaligen Ereignissen nennt man Sekundärtraumatisierungen.

b Unfälle und Stürze sind sogenannte Entwicklungstraumata, weil sie zu Fehlentwicklungen führen können.

c Entwicklungstraumata können zu schweren Bindungs- und Beziehungsstörungen führen.

d Traumata, die sich von einer Generation auf die nächste vererben, bezeichnet man als „intragenerational“.

e Transgenerationale Traumata überdauern nur zwei Generationen und werden dann von der DNA gelöscht.

\section{Auswahl 3}

a PTBS ist in der ICD-10 mit E 43.1 beziffert. b Im DSM IV findet sich zu PTBS eine Kriterienliste des amerikanischen Psychotherapieverbands.

c Gemäß Kriterienliste für Erwachsene mit PTBS im DSM IV sind die Sofortreaktionen der Betroffenen Wut und das Gefühl, ungerecht behandelt zu werden.

d Da sich das Nervensystem von Kindern noch entwickelt und somit ständig selbst heilt, können bei ihnen keine PTBS-Symptome auftreten.

e Gemäß Kriterienliste für Erwachsene mit PTBS im DSM IV schränkt das Syndrom Lebensführung und Gefühlsleben maßgeblich ein.

\section{Auswahl 4}

a Verstärktes Arousal macht es Betroffenen schwerer, sich zu entspannen.

b Menschen mit verstärktem Arousal können sich aufgrund der höheren Adrenalinausschüttung langfristig besser konzentrieren.

c Eine PTBS tritt spätestens nach 6 Wochen auf.

d Eine schwerwiegende Traumastörung ist DISNOS.

e Die Komplexe PTBS resultiert aus einer akuten PTBS.

\section{Auswahl 5}

a Die ICD-11 ist Januar 2020 in Kraft getreten.

b Die ICD-10 hat auch die anhaltende Trauerstörung als Diagnose aufgenommen.

c Die K-PTBS entspricht hinsichtlich ihrer Symptome eins zu eins der BorderlinePersönlichkeitsstörung.

d Im DSM V werden die Dissoziativen Identitätsstörungen ebenfalls beschrieben.

e Die DIS entstehen in der Regel durch einmalige Gewalterfahrungen.

\section{Auswahl 6}

a In der Dissoziation treten verschiedene Formen von Bewusstseinsspaltung auf.

b In der Dissoziation werden Gefühle und Körper intensiver wahrgenommen.

c Schockimplosion führt zu einer verstärkten Schmerzempfindung.

d Bei Todesgefahr blockiert das Nervensystem Dissoziationen stets.

e Das Überlebens-Kind ist Teilstruktur des Tabu-Kindes.

\section{Auswahl 7}

a Traumatisierte Menschen meiden Drogen in der Regel, denn sie fürchten Kontrollverlust.

b Zu den Zielen für traumatisierte Menschen gehört, von ihren übermächtigen Symptomen und Emotionen befreit zu werden.

c Mit Medikamenten können die Hypoarousals (Übererregtheit) herunterreguliert werden. d EMDR ist bei Traumata kontraindiziert.

e Die Kunsttherapie ist die erfolgreichste Therapieform für schwertraumatisierte Menschen.

\section{Auswahl 8}

a Schwere Traumata deaktivieren in der Regel die linke Gehirnhemisphäre.

b Das Modell des dreigliedrigen Großhirns stammt vom Hirnforscher Paul MacLarren.

c Das Stamm- oder Reptilienhirn ist der evolutionsgeschichtlich jüngste Hirnteil.

d Das limbische System ist unter anderem für die Verarbeitung von Emotionen zuständig.

e Zum limbischen System gehören Hypocampus und Amygdala.

Auswahl 9

a Die Amygdala ist Sitz des expliziten Gedächtnisses.

b Der Neokortex ist der zweitälteste Hirnteil.

c Im Fall einer Traumatisierung kann eine Hirnebene dominant werden.

d Bei einer dissoziativen Trennung der Gehirnhemisphären werden fragmentierte Traumaerinnerungen linkshemisphärisch verarbeitet.

e Deswegen werden wichtige Informationen bezüglich des Traumas im expliziten, aber nicht im impliziten Gedächtnis gespeichert.

\section{Auswahl 10}

a Der dorsale Vagus ist für den zwischenmenschlichen Kontakt zuständig.

b Auf extreme traumatische Bedrohung reagiert der Neokortex, indem er Hormone ausschüttet, die dem Körper signalisieren, dass er sich auf eine Abwehrreaktion vorbereiten muss.

c Erstarrung ist als physiologische Stressreaktion Kampf oder Flucht vorgeschaltet.

d In der Erstarrung nutzt das ZNS die Zeit, um sich kognitiv für Kampf oder Flucht zu entscheiden.

e Traumatisierte Menschen haben häufig mit Schuld- oder Schamgefühlen zu kämpfen. 


\title{
DHZ-Fortbildungspunkte - Antwortbogen
}

\author{
DHZ-Antworten
}

\section{Lernerfolgskontrolle}

Bitte nur eine Antwort pro Frage ankreuzen! Sie können in dieser

DHZ-Ausgabe 2 Fortbildungspunkte erzielen.

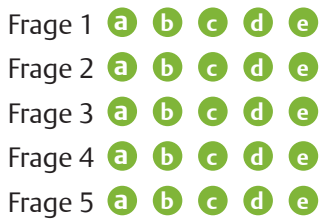

Frage 1 a b c d e

Frage 6 a b c d e

Frage 7 a b c d e

Frage 8 a b c (d) e

Frage 9 a b c d e

Frage 10 a b c d e

Angaben zur Person

Name, Vorname

Straße

PLZ/Ort

Feld für DHZ-Wertmarke/Abo-Nummer

Deutsche Post ${ }^{\circ}$ Porlag KG - Rüdigerstraße 14 - 70469 Stuttgart

2687 \# 12346 "\# $1 / 16^{*}$

2687 \# 12346 \# $1 / 16^{*}$

Elvira Mustermann Ihre Abonummer

Etikettstraße 11

Nichtabonnenten bitte hier DHZ-Wertmarke aufkleben. Abonnenten bitte Abonummer eintragen.

12345 Beispielhausen

\section{Erklärung}

$\square$ Ich versichere, dass ich die Beantwortung der Fragen selbst und ohne Hilfe durchgeführt habe.

Ort/Datum Unterschrift

Ihr Ergebnis

$\square$ Sie haben

Fragen richtig beantwortet. $\square$ Sie haben

DHZ-Punkte erworben. $\square$ Sie haben
nicht erfolgreich teilgenommen. 
obligatorisch. Mit dem Erhalt des Zertifikats verpflichten sich die zertifizierten Homöopathen, ihr Wissen ständig auf dem Laufenden zu halten. Regelmäßige Fortbildungen auf homöopathischem (30 Unterrichtseinheiten à 45 min pro Jahr) und schulmedizinischem Gebiet (8 Unterrichtseinheiten à 45 min pro Jahr) müssen alle zwei Jahre nachgewiesen werden, um die Zertifizierung aufrechtzuerhalten (Infos unter www.homoeopathie-zertifikat.de).

Die SHZ hat sich seit der Ausgabe DHZ 6/2008 entschieden, allen Zertifikatsinhabern die Chance der interaktiven Fortbildung anzubieten, um den Nachweis für die klinische Fortbildung zu erbringen. So können die Zertifikatsinhaber die Fortbildungspunkte der DHZ bei der SHZ als Nachweis für die klinische Fortbildung einreichen (maximal 4 Unterrichtseinheiten pro Jahr - das entspricht 4 Fortbildungspunkten der DHZ).

\section{Verbände und unabhängige Stiftung stehen für Qualität}

Das Entscheidende an diesen beiden Systemen ist, dass Teilnehmer nicht einfach Teilnahmebescheinigungen von Fortbildungen sammeln können, sondern dass eine Zertifizierungsstelle, hier ein Berufsverband bzw. eine Stiftung, für die Qualität der Fortbildung steht und diese überprüft und dokumentiert.

Das DHZ-Team unterstützt den Ansatz der regelmäßigen qualifizierten und dokumentierten Fortbildung. Die interaktive Fortbildung halten wir für ganz besonders geeignet, eine qualifizierte Fortbildung zu belegen, weil hier eine eindeutige Lernerfolgskontrolle besteht. Nur wer die Fragen richtig beantwortet, erhält auch seine Fortbildungspunkte.

\section{So geht's}

Sie finden in jeder DHZ-Ausgabe Fortbildungseinheiten. Diese können Sie - auch unabhängig von der Zertifizierung - regelmäßig nutzen, um Ihren Lernerfolg zu überprüfen und zu dokumentieren. Andererseits können Sie sich diese DHZ-Fortbildungspunkte auch für die Fortbildungszertifikate der genannten Verbände anerkennen lassen.

\section{Anerkennung der DHZ-Beiträge}

Die Vergabe der Fortbildungspunkte kann direkt durch die DHZ erfolgen. Pro Lerneinheit erhalten Sie 2 Fortbildungspunkte. Hierfür

- müssen 7 von 10 Fragen richtig beantwortet sein,

- muss der Antwortbogen vollständig ausgefüllt sein,

- muss im entsprechenden Feld des Antwortbogens eine DHZWertmarke aufgeklebt oder Ihre DHZ-Abonummer eingetragen sein. Die Abonummer finden Sie auf dem Adressaufkleber Ihres Heftes.

\section{Beantworten und abschicken!}

Zu einzelnen Beiträgen der DHZ gibt es in jeder Ausgabe Fragebögen (S. II), die Sie seit 2010 auch im Internet unter www.thieme-connect.de/ejournals/toc/dhz (auch als Nichtabonnent der DHZ) herunterladen und ausdrucken können. Schicken Sie uns einfach den ausgefüllten Antwortbogen (S. III). Wir prüfen Ihre Antworten und benachrichtigen Sie über Ihren Lernerfolg. Die Bescheinigung können Sie dann bei der zentralen Dokumentationsstelle des Fortbildungszertifikats für Heilpraktiker beim BDH und für das Homöopathiezertifikat bei der SHZ einreichen.

Die Teilnahme ist rückwirkend bis maximal ein Jahr nach Erscheinen der jeweiligen DHZ-Ausgabe möglich. Beachten Sie den Einsendeschluss auf dem Antwortbogen.

\section{Datenschutz}

Ihre Daten werden ausschließlich für die Bearbeitung dieser Fortbildungseinheit verwendet. Es erfolgt beim Verlag keine Speicherung der Ergebnisse über die für die Bearbeitung der Fortbildungseinheit notwendige Zeit hinaus. Die Daten werden nach Versand der Punktbescheinigungen anonymisiert. Namen- und Adressangaben auf den Antwortbögen dienen ausschließlich dem Versand der Punktbescheinigungen. Die Angaben zur Person dienen nur statistischen Zwecken und werden von den Adressangaben getrennt und anonymisiert verarbeitet.

\section{DHZ-Wertmarke für Nichtabonnenten}

DHZ-Wertmarken können beim Verlag erworben werden: 6erPack DHZ-Wertmarken; Preis 25,00 € inkl. MwSt.; Artikel-Nr. 902999.

\section{Bitte richten Sie Ihre Bestellung an: \\ Georg Thieme Verlag \\ KundenServiceCenter Buch \\ Postfach 3011 20; 70451 Stuttgart \\ Tel.: 0711/8931-900}

\section{Dieser Artikel ist online zu finden:}

http://dx.doi.org/10.1055/a-1195-7551

\section{TEILNAHME}

\section{Jeder kann mitmachen!}

Jeder Heilpraktiker soll die Möglichkeit zur Teilnahme an der Fortbildung erhalten. Deshalb ist die Teilnahme an der DHZ-Fortbildung nicht an ein Abonnement geknüpft. Dennoch sollten Sie im Besitz einer $\mathrm{DHZ}$ sein, denn alle im Fragebogen enthaltenen Fragen beziehen sich auf die Beiträge der jeweiligen Ausgabe. An diversen Heilpraktikerschulen finden Sie DHZ-Exemplare, die dort ausliegen. Darüber hinaus können Sie als Nichtabonnent der DHZ Einzelexemplare erwerben bei:

KundenServiceCenter Zeitschriften, Rüdigerstr. 14, 70469 Stuttgart, Tel.: 0711/8931-308. Für Abonnenten ist die Teilnahme kostenfrei. Nichtabonnenten können DHZ-Wertmarken erwerben. 